\title{
PERCEPÇÃO DOS ALUNOS DE MEDICINA COM RELAÇÃO À METODOLOGIA SOTL
}

\author{
ANDRÉIA ALMEIDA MENDES 1, HUMBERTO VINÍCIO ALTINO FILHO ${ }^{2}$, \\ RITA DE CÁSSIA MARTINS DE OLIVEIRA VENTURA ${ }^{3}$, REGINALDO \\ ADRIANO DE SOUZA ${ }^{4}$, MARCOS VINÍCIUS DE SOUZA ${ }^{\mathbf{5}}$, FERNANDA \\ COTA TRINDADE ${ }^{6}$, GLAUCIO LUCIANO ARAÚJO ${ }^{7}$, RENATA DE FREITAS \\ MENDES $^{8}$
}

\begin{abstract}
1 Doutora em Linguística pela Universidade Federal de Minas Gerais (UFMG), Coordenadora de Pesquisa e Extensão e professora do Centro Univeristário UNIFACIG. andreialetras@ yahoo.com.br 2 Mestre em Educação Matemática pela Universidade Federal de Ouro Preto. Professor e Analista Educacional no Centro Universitário UNIFACIG. humbertovinicio@ hotmail.com.

3 Doutora em Ciência da Informação pela Universidade Federal de Minas Gerais UFMG, pró-reitora e professora no Centro Universitário UNIFACIG dir.academica@ facig.edu.br

4 Mestre em Administração pela Faculdade de Estudos Administrativos de Minas Gerais FEAD, coordenador e professor no Centro Universitário UNIFACIG. reginaldoberbert@ hotmail.com 5 Doutor em Saúde Animal pela Universidade Federal de Uberlândia, coordenador e professor do Curso de Graduação em Medicina Veterinária no Centro Universitário UNIFACIG. mvscardoso@ yahoo.com.br. 6 Mestre em Arquitetura e Urbanismo pela Universidade Federal do Espírito Santo, coordenadora e professora no Centro Universitário UNIFACIG. fercotat@gmail.com.

7 Doutor em Engenharia Agrícola pela Universidade Federal de Viçosa UFV, coordenador e professor no Centro Universitário UNIFACIG. glaucio.araujo@sempre.facig.edu.br

8 Doutora em Ciências Biológicas pela Universidade Federal de Juiz de Fora, professora no Centro Universitário UNIFACIG. renatinhafmendes@gmail.com
\end{abstract}

\section{RESUMO}

Sabe-se da importância de se desenvolver atividades que levem o aluno a pesquisar e desenvolver o pensamento científico; quando essas habilidades se somam ao processo de ensino-aprendizagem, como uma ferramenta, este benefício se potencializa, o que contribui ainda mais para o desenvolvimento de habilidades e competências essenciais não só a vida acadêmica, como também à vida profissional. Com base nisso, objetivouse analisar a percepção dos alunos do $2^{\circ}$ de Medicina de um Centro Universitário da Zona da Mata Mineira com relação à metodologia SOTL, aplicada na disciplina de "Português Instrumental". Para a aplicação do método, optou-se pela escolha de artigos científicos ligados à temática da importância da comunicação para ampliar o potencial terapêutico de cuidado nas ações de saúde, uma vez que, durante o semestre, esta temática havia sido objeto de discussão e análise na disciplina. A aplicação do método SOTL seguiu as etapas propostas pelo método, tendo a duração de 2 horas. Ao final, foi aplicado um questionário de natureza quantitativa como forma de analisar a aceitação do método; a turma em questão possuía 45 alunos, sendo que, destes, 36 alunos 
responderam a este questionário. Registrou-se, então, que metade dos alunos consideraram o método como satisfatório e/ou excelente; $47,5 \%$ consideraram que o conteúdo da disciplina foi apreendido de forma mais significativa através do método e $41,7 \%$ afirmaram que a metodologia proporcionou a construção colaborativa do conhecimento e, por isso, deve ser recomendada a outros períodos e cursos. Dentre as habilidades desenvolvidas pelo método, a colaboração, a reflexão e a autonomia foram as mais recorrentes. Torna-se interessante destacar ainda que, apesar da DCN deste curso sinalizar a aplicação de metodologia ativa como obrigatória, observou-se a rejeição de parte dos alunos que ainda veem o método tradicional de ensino como o mais eficiente.

Palavras-chave: Metodologias Ativas, Ensino-Aprendizagem, SOTL, Medicina, DCN's.

\title{
PERCEPTION OF MEDICINE STUDENTS IN RELATION TO THE SOTL METHODOLOGY
}

\begin{abstract}
\end{abstract}
The importance of developing activities that lead the student to research and develop scientific thinking is known; when these skills are added to the teaching-learning process, as a tool, this benefit is increased, and contributes to the development of skills and essential competencies not only to academic life, but also to professional life. Based on this, the objective was to analyze the perception of students of the 2 nd half Medical School of a college in Wood Zone in Minas Gerais - Brazil, in relation to the SOTL methodology, applied in the discipline of "Instrumental Portuguese". For the application of the method, the choice was of scientific articles related to the theme of the importance of communication to expand the therapeutic potential of care in health actions, since, during the semester, this theme had been the subject of discussion and analysis in the discipline. The application of the SOTL method followed the steps proposed, lasting 2 hours. At the end, a quantitative questionnaire was applied as a way to analyze the acceptance of the method; the class is composed by 45 students, of which 36 responded to this questionnaire. It was registered, then, that half of the students considered the method as satisfactory and/or excellent; $47.5 \%$ considered that the content of the discipline was seized more significantly through the method and $41.7 \%$ affirmed that the methodology provided the collaborative construction of knowledge and, therefore, it should be recommended for other periods and courses. Among the skills developed by the method, collaboration, reflection and autonomy were the most recurrent. It is also interesting to note that, despite the NC (National Curriculum) of this course, signaling the application of active methodology as mandatory, it was observed the rejection of part of the students who still see the traditional teaching method as the most efficient.

Keywords: Active Methodologies, Teaching-Learning, SOTL, Medicine, DNC's. 


\section{INTRODUÇÃO}

A educação dos profissionais de saúde, em especial da Medicina, sempre se baseou, historicamente, no modelo flexeriano; este modelo enfatiza os aspectos meramente biológicos, fragmentando o saber e o descontextualizando da prática e, ainda, desconsiderando a realidade e as necessidades do Sistema Único de Saúde (SUS) no Brasil (SOBRAL, CAMPOS, 2012).

A adoção do modelo flexeriano, associado à metodologia de ensino tradicional, gera um sistema de ensino baseado em aulas meramente expositivas e uma série de avaliações e trabalhos meramente focados na memorização e na acumulação de conteúdos. Assim, além do conhecimento vir desassociado da prática, percebe-se uma mera exposição de informações, de forma conteúdista, por parte do docente que não se preocupa em desenvolver no discente habilidades e competências essenciais à sua atividade profissional (GLEADOW,HONEYDEW, FORD, BRONWYN, ABBOTT, 2005).

Tentando solucionar este impasse, o Ministério da Educação e Cultura (MEC), instituiu, por meio das Diretrizes Curriculares Nacionais (DCN's) brasileiras do curso de graduação de Medicina, em 2014, a adoção, na formação médica, de metodologias ativas de aprendizagem, colocando como uma das prerrogativas do curso a adoção de práticas pedagógicas que favoreçam ações transformadores, éticas e reflexivas, de forma a estimular a autonomia do estudante (BRASIL, 2014).

Considerando tudo isso, este artigo propõe analisar a percepção dos alunos do $2^{\circ}$ período de Medicina de um Centro Universitário da Zona da Mata Mineira, com relação à metodologia SOTL. Salienta-se que, apesar de ser a primeira aplicação dessa metodologia no curso, a adoção de metodologias ativas de aprendizagem por parte dessa instituição de ensino já ocorre desde 2012, não estando condicionada à DCN do MEC, tampouco à existência do próprio de curso de Medicina da instituição, uma vez que este curso teve início na instituição apenas em 2014. Ressalta-se, ainda, que os grandes propulsores dessa adoção foi o consórcio STHEM Brasil, do qual a instituição faz parte. Este processo de adoção de metodologias ativas ocorreu de forma gradual, através de grupos de estudo, cujos membros se tornaram multiplicadores entre os demais professores. Inicialmente, foram adotadas 6 metodologias ativas e os demais métodos foram sendo inseridos aos poucos, por meio das formações e capacitações do próprio 
consórcio e/ou através de pesquisas e congressos. O método Scholarship of Teaching and Learning (SOTL), em especial, foi apresentado durante a formação de professores do STHEM/Brasil, em Lorena, em maio de 2018, momento em que o método foi apresentado aos participantes.

\section{SCOLARSHIP OF TEACHING AND LEARNING (SOTL)}

O Scolarship of Teaching (SOTL) pode ser definido como uma investigação guiada que supõe uma reflexão crítica por parte de grupos de alunos (ANDERSEN, 2000). Ou ainda, um método de trabalho crítico, intelectual, que possui metas significativas de aprendizagem (KREBER, 2015). Para ser considerado um SOTL, o método necessita apresentar 3 características: ser público, suscetível de revisão crítica e avaliação e estar acessível aos membros da comunidade acadêmica (KREBER, 2000).

Segundo Boyer (1990), principal responsável pela expansão do método, o SOTL é uma maneira de investigação na qual o professor deve utilizar em suas práticas e que integra as seguintes atividades:

a) Investigação: momento em que se desenvolve o conhecimento de sua matéria e a melhor forma de ensinar;

b) Integração: etapa em que se estabelecem conexões entre as disciplinas, uma vez que o processo de conhecimento sobre a forma de ensinar requer um trabalho interdisciplinar entre diferentes áreas;

c) Aplicação da investigação e difusão: momento em que o professor deve aplicar o método e divulgar o processo de investigação de seu trabalho docente;

d) Prática docente e aprendizagem dos alunos: ocorre a análise da relação entre sua atividade de ensino e os resultados de aprendizagem dos alunos, sendo esta a principal etapa, uma vez que são os melhores resultados de aprendizagem que trarão sucesso à metodologia.

Em informe publicado, na Carnegie Foundation, intitulado Scholarship Reconsidered: Priorities of the Professoriate (1990), Boyer traz uma visão renovada do que é ser acadêmico, repensando o significado desta palavra e quais devem ser as prioridades dos professores. Com base nisso, o autor diz ser inútil falar em melhoria da 
qualidade do ensino se não forem reconhecidos os esforços que vários professores têm em melhorar esta qualidade, uma vez que não é fácil fazer com que os alunos aprendam, muito menos motivar professores para melhorarem suas práticas docentes por meio de inovações, por mais que elas sejam possíveis e necessárias. Alerta, ainda, que muitas instituições estão apenas preocupadas em desenvolver pesquisas e nas publicações oriundas delas, considerando o processo de aprendizagem dos alunos um perda de tempo. Este informe teve um impacto enorme na época, gerando reflexões até hoje.

Essas reflexões se tornam o foco principal do método, uma vez que a prática reflexiva é uma das principais chaves para uma boa prática educativa; assim, durante a aplicação do SOTL, os professores necessitam ter capacidade autocrítica tanto com relação às sua experiências quanto às suas intenções e princípios educativos, sendo essa capacidade que irá configurar a sua identidade profissional (KANE, 2002).

Dentro dessa metodologia, para que um professor ensine bem, ele precisa compartilhar suas experiências por meio de publicações em revistas e em eventos científicos; assim, todo o conhecimento obtido a partir de suas boas práticas e reflexões oriundas delas devem se tornar visíveis, pois só assim contribuirá para o enriquecimento e desenvolvimento do conhecimento pedagógico e para a profissionalização docente. Ao escrever e debater sobre o processo de ensino, toma-se consciência do processo de aprendizagem e das necessidades dos alunos e demandas de sua área (LUIS, SOBRINO, GONZÁLEZ-TORRES, 2013).

\section{METODOLOGIA}

Trata-se de uma pesquisa de abordagem quantitativa e natureza aplicada. Para tanto, optou-se pela aplicação de um Survey e, a partir dele, analisou-se a aceitação dos alunos do $2^{\circ}$ período de Medicina de uma Faculdade da Zona da Mata Mineira, Brasil, com relação à metodologia SOTL. Salienta-se que o método foi aplicado na disciplina de "Português Instrumental" e o tema trabalhado teve como enfoque a importância da comunicação para ampliar o potencial terapêutico de cuidado nas ações de saúde, temática esta que já havia sido objeto de discussão e análise na disciplina.

Para a aplicação do método SOTL, foram seguidas as seguintes etapas: a turma foi dividida em equipes de 6 alunos, cada grupo foi subdividido em 3 duplas, cada dupla 
recebeu um texto diferente, relacionado à temática proposta e, após a leitura, os alunos iniciaram uma reflexão em torno da temática, objetivos, propósito, resultados e conclusões de cada texto. Por meio destes dados, foi confeccionada, por cada equipe, uma grande tabela com as informações obtidas e, a partir delas, foi analisado um ponto comum em cada um dos itens analisados. Ao final, cada uma das equipes pôde analisar os trabalhos produzidos pelos outros colegas e fazer suas ponderações através de postits; a seguir, o grupo realizou um processo de autoavaliação de seu trabalho. O método foi aplicado na sala de metodologias ativas da instituição e teve duração de 2 horas.

FIGURA 1 - Tabelas produzidas pelos alunos ao final da aplicação da metodologia.

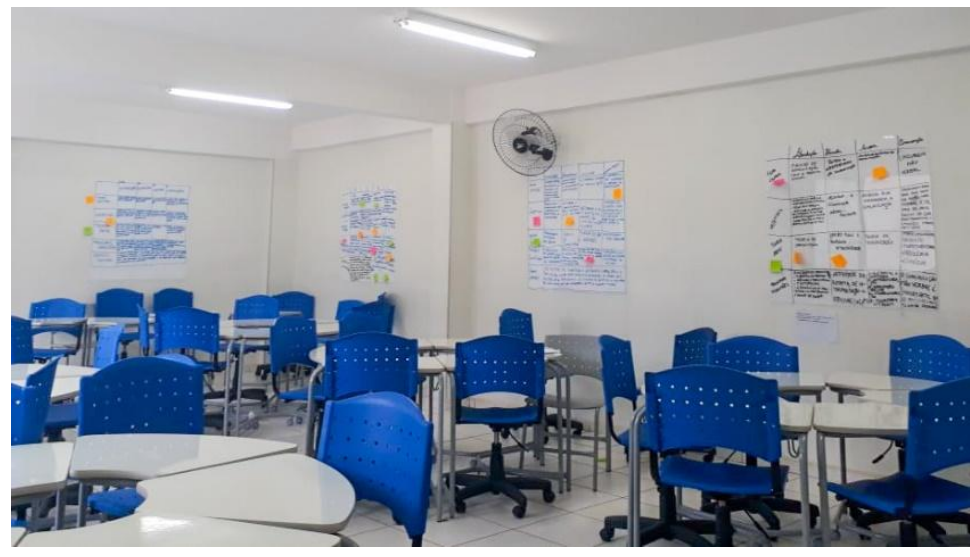

Fonte: Dados da pesquisa.

Ao final, aplicou-se um questionário on-line como forma de analisar a aceitação dos alunos com relação ao método, até então desconhecido pelos alunos; a turma em questão possuía 45 alunos e o questionário foi respondido por 36 alunos.

\section{RESULTADOS}

Após a aplicação dos questionários, os dados foram tabulados e os resultados serão apresentados em forma de gráficos nesta seção. A figura 1 apresenta o grau de aceitação dos alunos com relação ao SOTL.

FIGURA 2 - Grau de aceitação da metodologia ativa SOTL 


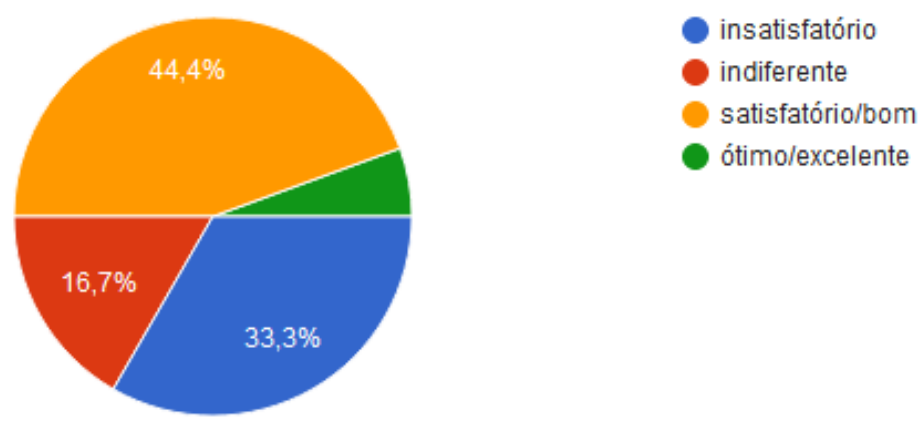

Fonte: Dados da pesquisa.

Observa-se, neste gráfico, que 50\% dos alunos consideraram a metodologia de forma positiva e os demais foram indiferentes ao processo e não a consideraram satisfatória. A figura 3 apresenta a percepção dos alunos com relação ao fato do trabalho com o método produzir aprendizagem mais significativa:

FIGURA 3 - Análise do método SOTL com relação à aprendizagem significativa
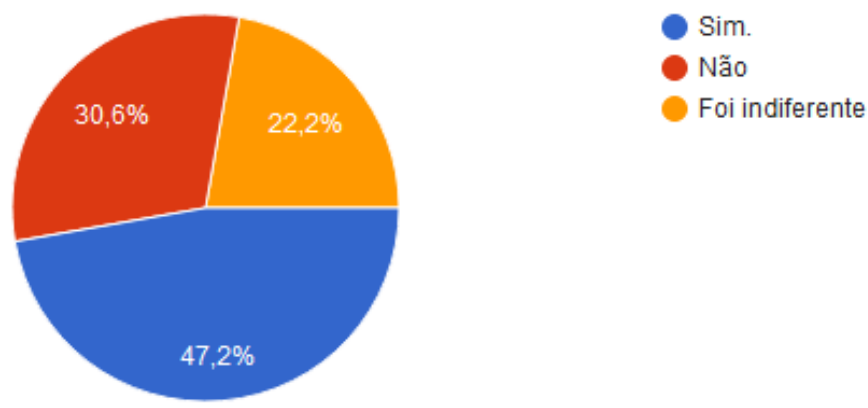

Fonte: Dados da pesquisa.

Observa-se que $47 \%$ dos alunos consideraram que o trabalho desenvolvido por eles durante a aplicação do método fez que com aprendessem o conteúdo de forma mais significativa. Em contrapartida, $30,6 \%$ desses alunos não consideraram que a aprendizagem se tornou mais significativa e $22,2 \%$ dos alunos julgaram-na indiferente.

Na sequência, os alunos avaliaram quais habilidades foram desenvolvidas ou potencializadas a partir da aplicação do método, obtendo-se o seguinte resultado: colaboração $(66,7 \%)$, planejamento $(38,9 \%)$, reflexão $(33,3 \%)$ e autonomia $(19,4 \%)$. 
A figura 4 apresenta os resultados apresentados quando os alunos foram indagados a respeito do fato de a aplicação do método ter gerado a construção colaborativa associada à aprendizagem, devendo, em função disso, o método ser aplicado em outros períodos do curso de Medicina e cursos da faculdade.

FIGURA 4 - Construção colaborativa associada à aprendizagem apresentada na disciplina e recomendação de sua aplicação a outros períodos e cursos.

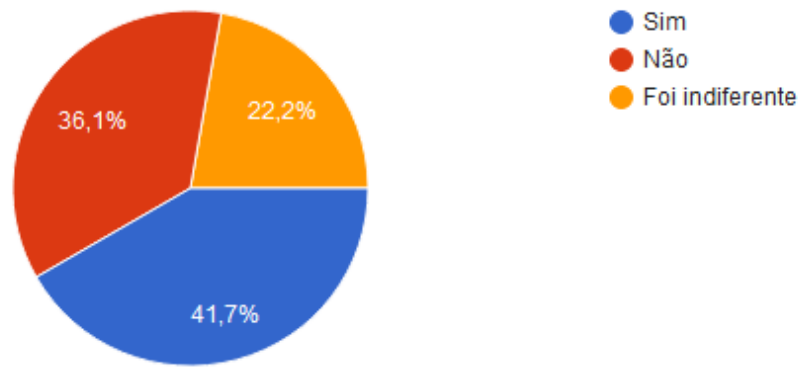

Fonte: dados da pesquisa.

Observa-se que $41 \%$ dos alunos consideraram o método fomentador de construção colaborativa associada à aprendizagem e que, em função disso, deve ser aplicado em outros períodos e cursos; em contrapartida, 36,1\% não concordam e $22,2 \%$ acreditam que a metodologia foi indiferente, ou seja, que qualquer outra metodologia tradicional surtiria o mesmo resultado.

Ao final do questionário, foi solicitado aos alunos que relatassem sua experiência com realização à aplicação do SOTL: os pontos positivos e negativos, o engajamento tido por eles, entre outras observações. Assim, destacam-se os seguintes resultados:

(1) "Foi satisfatório, possibilitou uma boa aprendizagem".

(2) "Forçou todos a ajudar no trabalho."

(3) "A comunicação com outras pessoas possibilita um melhor aprendizado".

(4) "Um bom método para o ensino dos períodos básicos, porém para períodos avançados, é necessário maior aprofundamento técnico". 
(5) "Pontos positivos: incentiva colaboração em grupo; ponto negativo: a conversa se torna alta e as avaliações são variadas, o que causa certa confusão, inclusive com o trânsito de pessoas na sala pequena".

(6) "Forçou todos a ajudar no trabalho".

(7) "Já conhecia a importância dos métodos citados".

(8) " Foi bom para o conhecimento"

(9) " Bom para aprender em conjunto, ruim que fica muito disperso".

(10) "Acho que aborda apenas alguns pontos do conteúdo e o tempo que temos para partilhar as informações e o conhecimento que temos não nos faz ser especialistas para expor com os colegas, o que nos deixa déficits com as matérias".

Pelas respostas dos alunos, percebe-se que a habilidade da colaboração, já apontada na figura 1 como a mais recorrente, foi novamente evidenciada nos comentários 3, 5, 6 e 9; além disso, o método foi apontado como estimulador de aprendizagem, uma vez que os "forçam" a ajudar (comentários 1, 2 e 6). O comentário 4 reflete que, na percepção do aluno, o método deve ser trabalhado apenas nas disciplinas iniciais do curso e os comentários 7 e 8 registram a satisfação dos alunos, sendo salientado, ainda, no comentário 7, o conhecimento e a importância desse tipo de metodologia por parte do aluno.

O comentário 5 relata ainda que o fato da sala ter sido pequena para o número de alunos que compõe a turma; o comentário realmente se fundamenta, uma vez que, para comportar todos os alunos, houve a necessidade de serem trazidas mais 5 cadeiras da sala ao lado.

Além desses resultados, foram apontados também comentários mais sucintos a respeito da metodologia, alguns positivos, a saber: "muito bom", "foi interessante", "gostei do método", "aprendi mais", "achei bom", "aula dinâmica", "achei legal e produtivo", "aprendi mais"; outros negativos e indiferentes, tais como: "confuso", "indiferente", "ok", "ajudou mais ou menos", "é chato", "atividade cansativa", "não gostei acho ruim", "dispersão".

Em algumas respostas, percebeu-se também a resistência de alguns alunos em romper com a metodologia tradicional de ensino: 
(11) "Otimização de tempo em aula, mas utilizar mais o método tradicional para não ter perca de tempo".

(12) "Não é a maneira objetiva de aprendizado".

(13) "Muito cansativo e não entendemos o motivo da atividade"

(14) "Não houve aprendizado de fato".

Além disso, alguns comentários refletiram o próprio comportamento de alguns alunos da turma, comportamento este já apontado como insatisfatório e atípico nos conselhos de classe da turma:

(15) "Com a turma falando alto, não dá para fazer trabalhos assim, infelizmente!"

(16) "A atividade se torna insatisfatória pela dispersão dos alunos".

(17) "A aceitação não foi pela sala toda, o que gerou cópias de trabalhos de alunos que não aderiram ao projeto".

(18) "Negativo - bagunça na sala"

Registra-se, nesses comentários, que a falta de adesão de alguns alunos realmente incomodou o restante dos alunos, prejudicando a execução do método em alguns momentos.

\section{DISCUSSÃO}

Após a apresentação dos resultados, podemos analisar a percepção dos alunos do $2^{\circ}$ período de Medicina de um Centro Universitário da Zona da Mata Mineira, Brasil, com relação à aplicação da metodologia SOTL na disciplina de "Português Instrumental".

Apesar de metade da turma ter aprovado a metodologia SOTL e considerar que o tipo de aprendizado apresentado por ela foi significativo, observa-se uma certa resistência de boa parte dos alunos no que diz respeito não apenas ao método SOTL como também às abordagens que fujam ao método tradicional de ensino. A adoção de metodologias ativas é considerada, por muitos autores, um grande desafio nos cursos de 
saúde pela dificuldade de rompimento com o sistema tradicional, flexeriano, conteudista e, principalmente, pela formação docente.

Com relação aos alunos, a forte presença da metodologia tradicional durante toda a trajetória acadêmica do aluno gera um estranhamento e até a incompreensão por parte dos alunos com relação ao ensino ativo (CÉSAR, GUIMARÃES GOMES, ROÇAS, SIQUEIRA-BATISTA, 2019). Realizar essa mudança no processo de ensinoaprendizagem é uma tarefa árdua, uma vez que ocasiona um ruptura não apenas no modelo tradicional de ensino, como também na própria forma de pensar a futura profissão:

reconhecendo essas necessidades, atualmente, muitas discussões apontam para a utilização de novas práticas pedagógicas e as instituições de ensino superior têm sido estimuladas a reconstruir seu papel social e valorizar a qualidade da assistência no trabalho em saúde, adotando tais inovações (SOBRAL, CAMPOS, 2012, p.209).

Devido a isso, torna-se necessário que as instituições de ensino, não só na área de saúde, forneçam aos alunos todas as informações necessárias para que entendam o papel do ensino ativo, bem como realizem a integração nas diferentes atividades desenvolvidas no decorrer do curso(CÉSAR, GUIMARÃES GOMES, ROÇAS, SIQUEIRA-BATISTA, 2019). Essas atividades devem promover a articulação entre ensino, pesquisa e assistência, tendo como foco a inovação e qualidade.

Assim sendo, torna-se necessário que os alunos compreendam que a sociedade atual exige inúmeras habilidades e competências do futuro profissional da área de saúde que não são adquiridas por meio do método tradicional, tais como: "a autonomia do aluno, a integração entre teoria e prática, uma visão crítica da realidade e o exercício do trabalho em equipe" (PAIVA, PARENTE, BRANDÃO E QUEIROZ, 2016).

Além disso, torna-se necessário, cada vez mais, que também se invista em um processo contínuo de capacitação com os professores para que possam compartilhar experiências, potencialidades e limites encontrados nas práticas, viabilizando-se sempre a discussão a respeito da melhoria desses processos (CÉSAR, GUIMARÃES, GOMES, ROÇAS, SIQUEIRA-BATISTA, 2019).

É certo que as metodologias ativas de ensino são muito importantes na formação do profissional da área médica, uma vez que têm contribuído para romper com o modelo tradicional de ensino, desenvolvendo habilidades e competências essenciais 
para a futura prática profissional, conforme ressaltado no próprio relato 4 . E também desenvolvem a integração entre teoria e prática, exercício do trabalho colaborativo, visão crítica da realidade e a autonomia do aluno (PAIVA, MEIRELLES, CURY, ALVES, 2016).

\section{CONCLUSÃO}

O objetivo deste estudo foi analisar a percepção dos estudantes de medicina acerca da metodologia SOTL, na disciplina de "Português Instrumental". De forma geral, o método foi bem aceito pelos discentes, apontando potencialidade no que se diz respeito ao trabalho colaborativo e ao desenvolvimento da comunicação.

Apesar disso, alguns estudantes ainda se encontram estacionados na metodologia tradicional pautada em aulas expositivas e na transmissão de conhecimentos que, apesar de deixarem o aluno numa zona de conforto, são obstáculos para o desenvolvimento de competências importantes para os profissionais da atualidade.

Dessa forma, é necessário que a aplicação desta metodologia e também de outras metodologias ativas seja difundida por todo o corpo docente do curso, também em outras disciplinas e conteúdos, como forma de habituar os alunos a um ambiente de protagonismo na aprendizagem, em que eles tenham papel ativo e possam desenvolver as habilidades e competências essenciais para seu futuro profissional.

\section{REFERÊNCIAS}

ANDERSEN, L.W. A usable, trans-disciplinary conception of scholarship. Higher Educationl-Research and Development, v. 19, n. 2, p. 137-153, 2000.

BRASIL. Ministério da Educação. Conselho Nacional de Educação. Câmara de Educação Superior. Resolução n. 3 de 20 de junho de 2014. Institui diretrizes curriculares nacionais do curso de graduação em Medicina e dá outras providências. Diário Oficial da União, Brasília, 23 jun. 2014.

BOYER, E. Scholarship Reconsidered. Washington, DC: The Carnie Foundation, 1990. 
CEZAR, P.H.N., GUIMARÃES, F.T., GOMES, A.P, ROÇAS, G., SIQUEIRA-

BATISTA, R. Transição paradigmática na educação médica: um olhar construtivista dirigido à aprendizagem baseada em problemas. Revista Brasileira de Educação

Médica. v. 34, n. 2, p. 298-303, 2010.

GLEADOW, R., HONEYDEW, M., FORD, A, BRONWYN, I., ABBOTT, K. New tools for a new age: An evolution or revolution in higher education? Research, v. 4, n. 1, p.1502, 2015.

KANE, How we teach the teachers: new ways to theoriza practice and practise theory. Prospect. v. 32, n. 3, 283-310, 2002.

KREBER, C. Reflection on teaching and the Scholarship of teaching: focus on science instructions. Higher Education-Research and Development. v. 50, n. 2, p. 523-359, 2015.

KREBER, C. How university teaching award winners conceptualise academic work: Some further thoughts on the meaning of scholarship. Teaching in Higher Education, v. 5, n. 1, p. 61-78, 2000.

LUIS, E. C.; SOBRINO, A.; GONZÁLEZ-TORRES, M.C. Scholarship of Teaching and Learning: un modelo de desarrollo profesional de los profesores universitarios. Revista Electrónica Interuniversitaria de Formación del Professorado, v. 16, n. 1, p.5-14, 2013.

SOBRAL, F. R., CAMPOS, C.J.G. Utilização de metodologia ativa no ensino e assistência de enfermagem na pordução nacional: revisão integrativa. Rev Esc Enferm USP, v. 46, n.1, p. 208-218, 2012.

PAIVA, M.RF.; PARENTE, J.R.F.; BRANDÃO, I.R.; QUEIROZ, A. H.B.

Metodologias ativas de ensino-aprendizagem: revisão integrativa. SANARE. v.15, n. 2, p. 145-153, 2016. 